Fallen von Silbernitrat mit Natriumacetat hergesteliten and nur durch Auswaschen gereinigten Produkt unternommen worden waren. In diesem konnte nur Natriumnitrat als Verunreinigung vorhanden sein, und als nun dem reinen Silberacetat eine Spur Salpeter zugesetzt wurde, trat die Oxydation wieder regelmăßig ein. Mehr Salpeter drückte jedoch die Ausbeute wieder herunter.

Das in kaltem Alkohol sehr schwer lösliche Pikrat wurde aus letzterem umkrystallisiert und schied sich dabei in derben, prismatischen Krystallen aus, die bei $160^{\circ}$ schmelzen.

$4.504 \mathrm{mg}$ Sbst: $7.368 \mathrm{mg} \mathrm{CO}, 1.252 \mathrm{mg} \mathrm{H} \mathrm{H}_{2} \mathrm{O}-4.72 \mathrm{mg}$ Sbst. : $0.7096 \mathrm{ccm}$ N (190, korr., $749 \mathrm{~mm})$.

$$
\begin{aligned}
& \mathrm{C}_{1}, \mathrm{H}_{30} \mathrm{~N}_{4} \mathrm{O}_{7} \text {. Ber. C 44.70, } \mathrm{H} \text { 3.10, } \mathrm{N} 17.28 \text {. } \\
& \text { Gef. - 44.62, } 3.11 \text {, } 17.28 \text {. }
\end{aligned}
$$

Aus dem reinen Pikrat wurde mit Alkali die Base freigemacht, mit Äther aufgenommen und destilliert. Sie ist eine wasserhelle Flüssigkeit von heftigem Pyridin-Geruch, raucht nicht an der Loft und siedet bei $121-122^{\circ}$.

Das Chlorhydrat ist sehr hygroskopisch; Zugeben von Platinchlorid zu der Lösung desselben erzeugt keine Fällung. Erst bei langsamem Eindunsten scheidet sich das Platinsalz in schönen, derben Krystallen aus, die beil $198^{\circ}$ schmelzen.

Durch Vergleich mit reinem $\alpha$-Picolin und dessen Salzen ergab sich die Identität der beiden Basen:

a-Picolin Basedurch Oxydation von Hexametbylen-imin Sdp. $128^{\circ}$ Sdp. 124-1250

$$
\begin{aligned}
& \text { Pikrat, Schmp. 1640 Pikrat, Schmp. 160" } \\
& \text { Misch-Schmelzpunkt der Pikrate } 162^{\circ} \text {. } \\
& \text { Pt-Salz, Schmp. } 195^{\circ} \quad \text { Pt-Salz, Schmp. } 198^{\circ} \\
& \text { Misch-Schmelzpunkt der Pt-Salze } 196^{\circ} \text {. }
\end{aligned}
$$

Auch unter dem Polarisationsmikroskop zeigten die beiden Basen dieselben Erscheinngen.

187. P. Friedlānder, Walter Herzog und G. V. Voß: Uber indigoide Farbstoffe der Phenanthren- und Inden-Reihe. [Ans d. Institut für Organ. Chemie d. Techn. Hochschule zu Darmstadt.] (Eingegangen am 4. April 1922.)

In einer Reihe früherer Arbeiten wurde eine Anzahl von unsymmetrischen indigoiden Farbstoffen beschrieben, weiche einerseits den Thionaphthen- oder Indol-Rest, andererseits den Komplex aromatischer Kohlenwasserstoffe des Benzols, Naphthalins, Anthracens und Acenaphthens (oder deren Substitutionsprodukte) enthalten und die als Indol (Thionaphthen)-benzol (Oxy-, Dioxy-benzol)-, - n a p h tha - 
lin (Oxy-, Amino-naphthalin)-, - anthracen (anthrol)-, acen aphthenindigo bezeichnet wurden. Verschiedene derselben finden praktische Verwendung als Küpenfarbstoffe wegen ihrer schönen Nuance und ihrer Beständigkeit.

Wir baben diese Gruppe vervollstăndigt durch Darstellung des Thionaphthen-phenanthren-indigos, sowie verschiedener Derivate des Indol- und Thionaphthen -indon-indigos. Für die Synthese derselben kamen die bereits bekannten Reaktionen zur Verwendung; 2-Thionaphthen-9'-phenanthren-indigo (I.) konte analog der Bildung des Thionaphthen-acenaphthen-indigos, durch Kondensation von Phenanthrenchinon mit 3-Oxy-thionaphthen erhalten werden.

I.

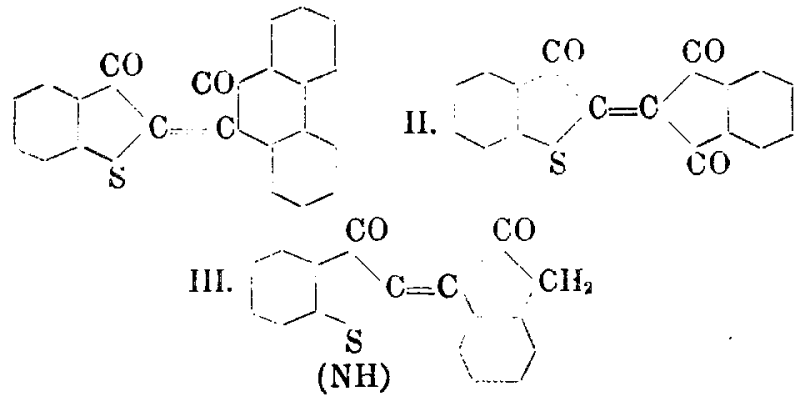

2-Thionaphthen-2'-indon-indigo (II.) entstebt aus 3-Oxythionaphthen-2-anilid und $\alpha, \gamma-$ Indandion, 2 -Thionaphthenresp. 2-Indol-1'-indon-indigo aus 3-0xy-thionaphthen-2-aniJid resp. $\alpha$-Isatin-anilid beim Erhitzen mit Essigsäure-anhydrid. Es wurde gefunden, daß sich letzteres bier wie in manchen anderen ávalogen Eällen durch Pyridin ersetzen läßt.

Die chemischen Eigenschaften der Farbstoffe sind die typischen dieser Gruppe: Küpenbildung und Aufspaltbarkeit durch Erhitzen mit starken Alkalien in zwei unsymmetrische Teile.

2-Thionaphthen- $9^{\prime}$ - phenanthren-indigo

Der Farbstoff scheidet sich schnell und in fast quantitativer Ausbeute in dunkelvioletten, fast sehwarzen Krystallen aus, wenn man eine siedende Lösung von $3.0 \times y-t h i o n a p h t h e n(3 \mathrm{~g})$ und Phenanthrenchinon $(4 \mathrm{~g})$ in Eisessig $(50 \mathrm{~g})$ mit einigen Tropfen konz. Salzsäure versetzt. Man saugt heißt ab, wäscht mit Alkohol und $\ddot{A}$ ther und krystallisiert aus heißem Nitro-benzol um, worin er sich reichlich mit tiefvioletter Farbe löst. $\mathrm{BnSO}_{4}$.

$0.2040 \mathrm{~g}$ Sbst.: $0.5770 \mathrm{~g} \mathrm{CO}_{2}, 0.0672 \mathrm{~g} \mathrm{H}_{2} \mathrm{O} .-0.1798 \mathrm{~g}$ Sbst.: $0.1274 \mathrm{~g}$

$$
\begin{aligned}
& \mathrm{C}_{22} \mathrm{H}_{12} \mathrm{O}_{2} \mathrm{~S} \text {. Ber. C 77.6, } \mathrm{H} 3.52, \mathrm{~S} 9.4 . \\
& \text { Gef. } 77.2,>3.68,=9.7 .
\end{aligned}
$$


Fast unlöslich in nieçriger siedenden Lösungsmitteln mit Ausvahme von Pyridin. $\mathrm{H}_{2} \mathrm{SO}_{4}$ löst unverändert mit violetter Farbe. Alkalische Reduktionsmittel geben eine gelbe Küpe, aus der Textilfasern trübe-violett gefärbt werden. Die sehr oxydable, nicht krystallisiert erhaltene Leukoverbindung gibt beim Erwärmen mit Essigsăure-anhydrid ein Diacetylderivat, das aus Xylol in schwach grünlichen Krystallen erhalten wurde.

$0.1682 \mathrm{~g}$ Sbst.: $0.4510 \mathrm{~g} \mathrm{CO}_{2}, 0.0538 \mathrm{~g} \mathrm{H}_{2} \mathrm{O}$.

$\mathrm{C}_{26} \mathrm{H}_{18} \mathrm{O}_{4} \mathrm{~S}$. Ber. C 73.24, H 4.22 .

Gef. * $73.12,4.51$.

Der Farbstoff ist außerordentlich widerstandsfähig gegen Alkalien und wird davon erst bei Temperaturen verāndert, bei welchen der vermutlich intermediär gebildete 9-Oxy-phenanthren-10-aldehyd weitere Zersetzungen erleidet. Zum Vergleich wurde das Verhalten des ähnlich gebauten Thionaphthen-acenaphthen-indigos (Thioindigo-Scharlach 2 G) (IV.) untersucht, der sehr viel leichter im Sinne nachstehender Formeln zu Thio-salicylsäure (V.) und (enol-)2Acenaphthenon-1-aldehyd (VI.) aufgespalten wird. Man erhitzt am besten mit der 10-fachen Menge konz. alkobolischer Kalilauge

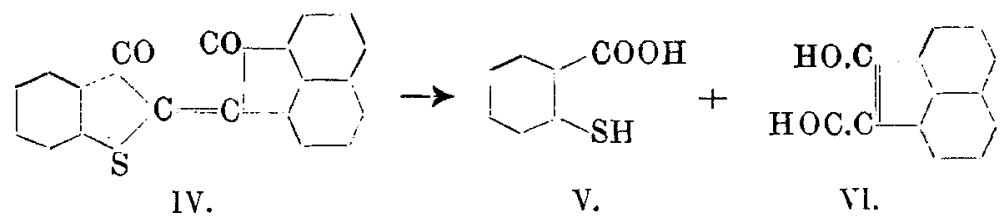

(im Ölbad) auf $90-100^{\circ}$, wobei dex Farbstoff zunächst mit dunkelgrüner Farbe in Lösung geht, aus der sich allmählich an den Gefäßwänden orangerote Nadeln eines wasserlöslichen Kaliumsalzes abscheiden, die über Asbest abfiltriert und mit alkoholischer Kalilauge ausgewaschen werden. Säuren scheiden aus der wäbrigen Lösung den freien Aldehyd in fast farblosen Flocken aus, die bei $163^{\circ}$ schmelzen, leicht in Alkohol und Essigsäure, schwer in Benzol, Ligroin und Äther, fast unlöslich in kaltem Wasser sind und aus sehr viel heißem Wasser in krystallinischen, schwach grünlich gelben Flocken erhalten werden können.

$$
\begin{aligned}
0.1500 \mathrm{~g} \text { Sbst.: } 0.4362 \mathrm{~g} \mathrm{CO}_{2}, 0.0640 \mathrm{~g} \mathrm{H}_{2} \mathrm{O} . \\
\mathrm{C}_{13} \mathrm{H}_{8} \mathrm{O}_{2} \text {. } \\
\\
\text { Ber. C 79.6, } \mathrm{H} \text { 4.0. } \\
\text { Gef. 79.4, 4.6. }
\end{aligned}
$$

Die ziemlich zersetzliche Verbindung wird schon von Soda gelöst. Eisenchlorid färbt die verd. alkoholische Lösung rot. Auf Zusatz von Phenylhydrazin zu der mit etwas Essigsăure angesăuerten Lösung in Alkohol scheiden sich schöne, gelbe Nadeln eines Phenyl-hydrazons ab, die, aus verd. Alkohol umkrystallisiert, bei $170^{\circ}$ unter Zersetzung schmelzen. 
$0.1532 \mathrm{~g}$ Sbst.: $14.8 \mathrm{ccm} \mathrm{N}\left(17^{\circ}, 747 \mathrm{~mm}\right)$.

$\mathrm{C}_{19} \mathrm{H}_{14} \mathrm{ON}_{2}$. Ber. $\mathrm{N}$ 9.9. Gef. $\mathrm{N}$ 10.3.

Beim Schütteln der soda-alkalischen Lösung des Aldehyds mit Dimethylsulfat scheidet sich ein Methyläther ab, der aus verd. Alkohol in schönen, farblosen Nadeln krystallisiert,

$0.1532 \mathrm{~g}$ Sbst.: $0.4508 \mathrm{~g} \mathrm{CO}_{2}, 0.0732 \mathrm{~g} \mathrm{H}_{2} \mathrm{O}$.

$$
\begin{array}{ll}
\mathrm{C}_{14} \mathrm{H}_{10} \mathrm{O}_{2} & \text { Ber. C 80.0, H } 4.8 . \\
\text { Gef. } & 80.4 \text {, 5.3. }
\end{array}
$$

und wie der Oxyaldehyd mit Phenyl-hydrazin ein in schönen, gelben Nadeln krystallisierendes Phengl-hydrazon (aus verd. Alkohol) vom Schmp. $172^{\circ}$ liefert.

$0.2000 \mathrm{~g}$ Sbst.: $16.6 \mathrm{~cm} \mathrm{~N}\left(15^{0}, 764 \mathrm{~mm}\right)$.

$\mathrm{C}_{20} \mathrm{H}_{16} \mathrm{~N}_{2}$ O. Ber. N 9.3. Gel. N 9.7.

\section{2-Indol-1'-indan-indigo (III.)}

Als Ausgangsprodukt für die nachstehenden Synthesen diente das 2-Indanon ( $\beta$-Keto-hydrinden), daß wir uns auf dem von $A$. Spieker ${ }^{1}$ ) angegebenem Wege darstellten.

Das erforderliche Inden stand uns in größerer Menge in 80-proz. Reinheit seitens der Gesellschaft für Teerverwertung, Duisburg-Meiderich, zur Verfügung, der wir auch an dieser Stelle unseren besten Dank aussprechen möchten.

Wir fanden, daß sich die Verbindung mit reaktionsfähigen Aldebyden nicht zu gleichen Molekülen, sondern im Verhältnis 1:2 kondensiert. Versetzt man eine warme Lösung von $\beta$-Indanon und $p$-Nitro-benzaldehyd in Eisessig mit einigen Tropfen konz. Salzsäure, so scheidet sich eine schwer lösliche, gelbe Verbindung von hohem Schmelzpunkt ab, die aus wenig Nitro-benzol in kleinen, gelben Nadeln erhalten wurde.

$0.1352 \mathrm{~g}$ Sbst.: $0.3454 \mathrm{~g} \mathrm{CO}_{2}, 0.0429 \mathrm{~g} \mathrm{H}_{2} \mathrm{O} .-0.2269 \mathrm{~g}$ Sbst.: $14.1 \mathrm{ccm}$ $\mathrm{N}(220,758 \mathrm{~mm})$.

$$
\begin{aligned}
& \mathrm{C}_{16} \mathrm{H}_{\mathrm{i}_{1}} \mathrm{O}_{3} \mathrm{~N} \text {. Ber. C 72.45, H 4.15, N 5.27. } \\
& \mathrm{C}_{23} \mathrm{H}_{14} \mathrm{O}_{5} \mathrm{~N}_{2} \text {. } \gg 69.31,>3.52,-7.05 \text {. } \\
& \text { Gef. • 69.65, } 3.55 \text {, } 6.99 \text {. }
\end{aligned}
$$

In derselben Weise wurde aus $\beta$-Indanon und $p-O x y-b e n z-$ aldehyd ein Kondensationsprodukt erhalten, das aus Eisessig in gelben, glänzenden Nadeln vom Schmp. ca. $265^{\circ}$ unter Zersetzung krystallisiert. Lőslich in Natronlauge mit orangeroter, in konz. Schwefelsäure in der Kälte ohne Veränderung mit blauvioletter Farbe, schwer löslich in Alkohol, Äther und Benzol.

1) B. 26, 1541 [1893]; vergl. F. Heasler und H. Schiefler, B. 32, 29 [1899]. 
$0.1305 \mathrm{~g}$ Sbst: $0.3872 \mathrm{~g} \mathrm{CO}_{9}, 0.0565 \mathrm{~g} \mathrm{H}_{8} \mathrm{O}$.

$\mathrm{C}_{23} \mathrm{H}_{16} \mathrm{O}_{3}$. Ber. C 81.15, $\mathrm{H}$ 4.74.

Gef. - 80.92, 4.84 .

Hiernach schien es nicht ausgeschlossen, daß sich $\beta$-Indanon auch mit 2 Mol. $\alpha$-Isatinchlorid usw. zu einem di-indigoiden Farbstoff würde vereinigen lassen. Dies ist aber nicht der Fall, es entstehen nur Farbstuffe rom Typus III, selbst bei Anwendung von überschüssigem Isatinchlorid usw. 2-Indol-1'indan-indigo wird erbalten sowohl aus $\beta$-Indavon $+\alpha$-Isatinchlorid in Benzol, wie aus $\beta$-Indanon $+\alpha$-Isatin-anilid in der 4-fachen Menge Essigsäureanhydrid und scheidet sich aus diesen Lösungen schon bei gelindem Erwärmen in blauvioletten, rosettenförmig angeordneten Krystallen aus, die mit Alkohol und Äther gewaschen und aus siedendem Xylol umkrystallisiert wurden.

$0.2182 \mathrm{~g}$ Sbst.: $0.6235 \mathrm{~g} \mathrm{CO}_{2}, 0.0812 \mathrm{~g} \mathrm{H}_{2} \mathrm{O} .-0.2037 \mathrm{~g}$ Sbst.: $9.8 \mathrm{ccm}$ N $\left(19^{\circ}, 755 \mathrm{~mm}\right)$.

$$
\begin{array}{ll}
\mathrm{C}_{17} \mathrm{H}_{11} \mathrm{O}_{2} \mathrm{~N} . & \text { Ber. C 78.12, H } 4.24, \mathrm{~N} 5.38 . \\
& \text { Gef. } 77.92, \bullet 4.16, * 5.47 .
\end{array}
$$

Der Farbstoff ist in niedriger siedenden Lösungsmitteln kaum löslicb, ziemlich leicht in Nitro-benzol und siedendem Xylol mit blauroter Farbe. Trocken erhitzt, sublimiert er (unter partieller Zersetzung) in rotvioletten Dämpfen, konz. Schwefelsäure löst obne Verānderung mit olivgrünen, rauchende unter Sulfurierung mit blaugrüner Farbe. Unlöslich in Alkohol wie in wäBriger Natronlauge wird es von alkoholischer Natronlauge beim Erwärmen mit intensiv violetter Nuance als Farbstofl-alz ${ }^{1}$ ) aufgenommen und aus der Lösung durch Säuren wieder unverändert abgeschieden. Eine Aufspaltung durch Alkalien erfolgt auch bei böherer Temperatur nur sehr schwierig. Alkalisches Hydrosulfit gibt eine hellgelbe Küpe mit rotvioletter Blume, aus der namentlich Wolle sehr intensiv dunkelbordeaux gefärbt wird ${ }^{2}$ ).

Die Zusammensetzung des Farbstoffs wurde bestätigt durch die Analyse seines Dibromderivats aus Dibrom-isatinchlorid und Indanon, das in Lösungsmitteln etwas löslicher ist, in Nuance und Verbalten im übrigen dio größte Äbnlichkeit zeigt. $\triangle g B r$.

$0.1538 \mathrm{~g}$ Sbst.: $0.2728 \mathrm{~g} \mathrm{CO}, 0.03095 \mathrm{~g} \mathrm{H}, 0$. $-0.1202 \mathrm{~g}$ Sbst.: $0.1088 \mathrm{~g}$

$$
\begin{array}{ll}
\mathrm{C}_{17} \mathrm{H}_{9} \mathrm{O}_{2} \mathrm{NBr}_{2} . & \text { Ber. C 48.68, H 2.16, } \mathrm{Br} 3817 . \\
& \text { Gef. } \quad 48.36, \geqslant 2.24,>38.36 .
\end{array}
$$

1) vergl. P. Friedländer, B. 41, 1035 [1908].

ग) D. R. P. 227862, 231542 (Frdl. 10, 538, 540).

Beriohte d. D. Chem. Gesellschaft. Jahrg. IV. 
2-Thionaphthen-1'-indan-indigo (III.).

Eine Lösung von Thionaphthenchinon-2-anil (3.4 g) und $\beta$-Indanon (2 g) in wenig Essigsäure-anhydrid oder Pyridin erstarrt nach kurzem Erhitzen zu einem dicken Brei rotvioletter Krystalle, die nach dem Absangen mit Alkohol und Äther gewaschen und aus Nitro-benzol umkrystallisiert werden. $\mathrm{BaSO}_{4}$.

0.1655 g Sbst.: $0.4433 \mathrm{~g} \mathrm{CO}_{2}, 0.0499 \mathrm{~g} \mathrm{H}_{2} \mathrm{O}$. $-0.2171 \mathrm{~g}$ Sbst.: $0.1868 \mathrm{~g}$

$$
\begin{array}{r}
\mathrm{C}_{17} \mathrm{H}_{10} \mathrm{O}_{2} \mathrm{~S} \text {. Ber, C 73.35, H } 362, \mathrm{~S} 11.53 . \\
\text { Gef. } 73.05 \text {, } 3.37, \text { 11.82. }
\end{array}
$$

Fast unlöslich in den gebräuchlicben niedriger siedenden Lösung ${ }^{-}$ mitteln, leicht in heißem Nitro-benzol mit roter, in $\mathrm{H}_{2} \mathrm{SO}_{4}$ (unverändert) mit trüb grüner Farbe; färbt aus der gelben Hydrosulfitküpe Textilfasern wesentlich rotstichiger als der entsprechende Iodol-Farbstoff.

Nach demselben Verfahrea wurde aus molekularen Mengen von Thionaphtheńchinon-2-anil und $\alpha, \gamma$-Indandion

$$
\text { 2-Thionaphthen-2'-indon-indigo (II.) }
$$

erhalten, dessen Indol-Analogon bereits früber beschrieben wurde ${ }^{\mathbf{1}}$ ). Die Vereinigung erfolgt beim Erwärmen beider Komponenten in Essigsäure-anhydrid oder Pyridin leicht und fast quantitativ. Der Farbstoff scheidet sich in feinen, rotvioletten Nadeln aus, die in Chloroform und Eisessig ziemlich leicht, in den gebräuchlichen niedriger siedenden Lösungsmitteln schwer löslich sind. Ein aus Xylol umkrystallisiertes Pridukt ergab: $\mathrm{BaSO}$.

$0.1326 \mathrm{~g}$ Sbst: $0.3398 \mathrm{~g} \mathrm{CO}_{2}, 0.0316 \mathrm{~g} \mathrm{H}_{2} \mathrm{O} .-0.1225 \mathrm{~g}$ Sbst.: $0.0936 \mathrm{~g}$

$$
\begin{array}{ll}
\mathrm{C}_{17} \mathrm{~B}_{8} \mathrm{O}_{3} \mathrm{~S} . & \text { Ber. } \mathrm{O} \text { 69.83, H 2.76, S } 10.97 \text {. } \\
& \text { Gef. } \$ 69.79, \gg 2.67, \$ 10.5 \mathrm{l} .
\end{array}
$$

Konz. Schwefelsäure löst intensiv smaragdgrün, 10-proz. Natronlauge beim Erwärmen gelb, zunächst obne Aufspaltung in Thiosalicylsäure und Indandion aldehyd ${ }^{\text {) }}$, die erst bei energischer Einwirkung eintritt. Die Reduktion mit Soda und Hydrosulfit verläuft in zwei Phasen; es entsteht zuerst eine braunstichig violette Lösung, aus der sich dunkelviolette Kryställchen eines Farbstoffsalzes abscheiden, deren Farbstoffsäure, durch Essigsäure in Freiheit gesetzt, sich in Essigärher violett löst und durch alkalisches Blutlaugensalz zu dem ursprünglichen Farbstoff regeneriert wird. Bei weiterer Reduktion wird die Küpe sehr viel heller und braun. Vermutlich ist bei der Reduktion auch die zweite CO-Gruppe des Indandions beteiligt. Bei trocknem Erhitzen sublimiert der Farbstoff in orangegelben Dämpfen.

1) P. Friedländer und H. Felix, M. 31, 55. 